\title{
Evaluación Informatizada de la Atención para Niños de 7 a 11 Años: El DiViSA-UAM y el TACI- UAM
}

\section{Computerized Assessment of Attention for Children from 7 to 11 Years Old: DiViSA-UAM and TACI-UAM}

\author{
M. a Ángeles Quiroga Estévez \\ U. Complutense de Madrid, España \\ Alejandra Montoro \\ U. Autónoma de Madrid, España
}

Agustín Martínez-Molina

U. Autónoma de Madrid, España
José Santacreu Mas

U. Autónoma de Madrid, España

Pei Chun Shih

U. Autónoma de Madrid, España

Resumen. El objetivo de este estudio ha sido elaborar dos test informatizados que midan los componentes de la atención que permiten caracterizar a los niños diagnosticados TDAH. Para su elaboración se han tenido en cuenta los estudios sobre desarrollo de la atención así como las críticas recibidas por los instrumentos de evaluación disponibles, en un claro intento por superarlas. Ambos test se administraron a 423 escolares (de $2^{\circ}$ a $6^{\circ}$ ) de 7 a 11 años de edad. Los datos obtenidos muestran el patrón de desarrollo de cada uno de los índices que aportan las pruebas: eficacia general de la atención, inexactitud, olvidos, organización de la ejecución y distracción-precipitación. Los índices de fiabilidad de estos índices se hallan en el rango 0.77 a 0.96 que se considera satisfactorio. En la discusión se reseña la utilidad que pueden tener ambos test para la valoración del perfil atencional en el niño, sea con un objetivo académico o clínico.

Palabras clave: desarrollo de la atención, pruebas informatizadas, escolares, hiperactividad, impulsividad.

\begin{abstract}
The main goal of this study was to design two computerized tests to assess attention components relevant to children diagnosed with ADHD. For this purpose, previous developmental studies have been considered, and criticisms raised of previously employed tests. Both tests were administered to 423 school children from 7 to 11 years old (from $2^{\text {nd }}$ to $6^{\text {th }}$ grade). The data obtained show the developmental pattern of each index provided by each test: general efficiency of attention, inaccuracy, omissions, organization, and distraction- precipitation. Reliability indexes are satisfactory for both tests, ranging from 0.77 to 0.96. The discussion includes the utility these tests could have for assessment of the individual attentional profile in children, be it for academic or clinical purposes.

Keywords: development of attention, computerized tests, school children, hyperactivity, impulsivity.
\end{abstract}

La correspondencia sobre este artículo dirigirla a M. ${ }^{a}$ Ángeles Quiroga, Facultad de Psicología, Campus de Somosaguas, UCM 28223 Madrid. E-mail: m.angelesquiroga@psi.ucm.es
Este estudio se ha desarrollado en el seno del Grupo de Investigación Psi+D-UAM sin cuyo apoyo económico y técnico no hubiera sido posible llevarlo a cabo. Agrademos a los equipos directivos de los colegios Buero Vallejo y Príncipe Felipe de San Sebastián de los Reyes y al colegio Alarcón de Pozuelo de Alarcón, todos ellos de la CA de Madrid, su colaboración en la recogida de datos. 


\section{Introducción}

Desde un punto de vista psicológico, la atención se considera un concepto multidimensional cuyos componentes varían en denominación aunque existe un cierto consenso acerca de los tres más relevantes: atención sostenida, referido a mantener la atención en alerta, medido a través del rendimiento en tareas monótonas sencillas; atención selectiva, referido a focalizar la atención desechando los elementos distractores e irrelevantes, concentrándose en la tarea y control de la ejecución, referido a inhibir la tendencia a responder de manera automática e irreflexiva (Castillo, 2009; Lehman, Naglieri y Aquilino, 2010; Tudela, 1992).

Estos tres componentes están incluidos en los criterios diagnósticos utilizados para la valoración del trastorno por Déficit de Atención con o sin Hiperactividad (TDAH, DSMIV-TR, 2002). En estos criterios se resalta que la ejecución del niño debe mostrar un patrón persistente de desatención y/o hiperactividad que sea más frecuente de lo observado en niños de un nivel de desarrollo similar. Por lo tanto es necesario conocer el patrón de desarrollo de los tres componentes mencionados.

Los estudios sobre el desarrollo de estos tres aspectos (atención sostenida, atención selectiva y funciones ejecutivas) son relativamente recientes (Korkman, 2001) y heterogéneos en los modelos teóricos de partida y por tanto en los instrumentos de evaluación (Kanaka, Matsuda, Tomimoto, Noda, Matsushima et al., 2008; Klenberg, Korkman y Lahti-Nuutila, 2001; Lehman, Naglieri y Aquilino, 2010; Lin, Hsiao y Chen, 1999; Rueda, Fan, McCandliss, Halparin, Gruber, Pappert y Posner, 2004).

Rueda, Fan, McCandliss, Halparin, Gruber, Pappert y Posner (2004) estudiaron el desarrollo de las funciones de la atención basándose en la teoría de las tres redes atencionales (modelo de Posner y Petersen, 1990): a) orientación - focalización, b) vigilancia - mantenimiento de la atención y c) control ejecutivo de la actuación. Evaluaron a un grupo de niños de 6 a 9 años (6 niños y 6 niñas de cada edad) y a otro de 10 años que compararon con adultos. Utilizaron la batería ANT (Attention Network Test, Fan, McCandliss, Sommer, Raz y Posner,
2002) adaptada para niños por Berger, Jones, Rothbart y Posner, (2000). Esta batería incluye una sola tarea de reducida duración (25 minutos, máximo) presentada y respondida informáticamente. Los resultados obtenidos mostraron que la orientación focalización no cambia en el rango de edad estudiado (luego ya está desarrollada), la vigilancia (atención sostenida) mejora hasta y por encima de los 10 años, y la función ejecutiva se mantiene estable a partir de los 7 años. Además, las tres redes atencionales son relativamente independientes.

Klenberg, Korkman y Lahti-Nuutila (2001) utilizaron los datos de la estandarización al finlandés del NEPSY que es una batería neuropsicológica. Analizaron únicamente los 10 sub-test referidos a atención y a función ejecutiva. En la estandarización participaron 400 niños de 3 a 12 años, de ambos sexos y procedentes de familias de los tres niveles educativos presentes en el país. De las 10 tareas analizadas, las que se refieren a atención, evalúan: (a) inhibición motora; (b) atención selectiva auditiva; (c) atención sostenida auditiva; (d) búsqueda visual y (e) atención selectiva visual. Los resultados obtenidos mostraron: (1) efectos de la edad en todas las tareas; (2) interacción de la edad y el sexo sólo para una de las tareas de inhibición motora, de modo que las niñas realizaban mejor la tarea entre los 3 y los 5 años mientras que a partir de los 6 los niños la realizaban igual que las niñas; (3) un patrón evolutivo diferente para las tareas de inhibición motora y las de atención propiamente dicha, de forma que en las primeras crece hasta los 6-7 años y se mantiene estable al nivel de los 12 años, mientras que en las de atención aumenta hasta los 10 años y se mantiene estable al nivel de los 12 años. Los autores concluyeron que el desarrollo de la atención procede secuencialmente: primero maduran la inhibición motora y el control de impulsos y después la atención sostenida y selectiva.

Lehman, Naglieri y Aquilino (2010) utilizaron los datos de la estandarización del Sistema de Evaluación Cognitiva (CAS, Cognitive Assessment System). El Sistema CAS evalúa planificación, atención, procesamiento simultáneo y procesamiento sucesivo que son los componentes de la teoría PASS (Naglieri y Das, 1997). La muestra constaba de 2200 niños de 5 a 17 años de edad, representativos de la 
población estadounidense. Los autores analizaron los cambios asociados a la edad, así como la tasa de desarrollo entre edades próximas, para la Escala de Atención del CAS. Esta escala consta de tres subtest que miden: atención selectiva, atención sostenida + memoria operativa y resistencia a la interferencia. Los resultados mostraron que el patrón de cambio desde los 5 a los 15 años fue continuo y semejante para las tres tareas ya que la tasa de cambio, (calculada como la $d$ de Cohen, 1988), entre grupos de edad adyacente, fue semejante.

El desarrollo de la atención en niños también se ha estudiado a través de una tarea experimental clásica de atención: el CPT (Continuous Performance test). Los test tipo CPT, de amplia tradición en el estudio de la atención en adultos, están basados en la teoría de detección de señales y requieren pulsar una tecla ante la aparición de un estímulo preestablecido. Se trata por tanto de una tarea de vigilancia (atención sostenida) de discriminación o búsqueda, normalmente, visual. Lin, Hsiao y Chen, (1999) utilizaron el CPT para el estudio del desarrollo de la atención en niños, usando como estímulos números del 0 al 9 en dos versiones, simple (pulsar el 9) y compleja (estímulos desdibujados y pulsar el 9 si va precedido de un 1). Cada número se presentaba durante 50 milisegundos, a razón de un número por segundo. El estímulo diana (el n ${ }^{\circ}$ 9) sólo aparecía el $10 \%$ de las veces. Analizaron por separado el desarrollo de los aciertos y los errores en 341 niños de 6 a 15 años de edad, seleccionados aleatoriamente entre las escuelas de Chinshaw al norte de Taipei. Además de los aciertos y errores calcularon los índices d' (sensibilidad, que indica la capacidad para diferenciar entre el estímulo preestablecido y los demás) y $\beta$ (criterio de respuesta, que indica la tendencia a responder sólo cuando aparece el estímulo clave -criterio alto de respuesta- o responder a la mayor parte de los estímulos -criterio bajo de respuesta-), derivados ambos de la teoría de detección de señales. Los resultados obtenidos mostraron que la tasa (o la proporción) de aciertos y d' aumentan con la edad, resultado congruente con la hipótesis de que la inhibición se desarrolla durante los años de escuela primaria y con los resultados obtenidos por Klenberg et al. (2001) con el NEPSY. En paralelo, los errores de comisión o falsas alarmas disminuye- ron con la edad. $\beta$ no aumentó ni disminuyó con la edad en el CPT más sencillo, pero sí en el complejo. $\beta$ fue menor en los niños más pequeños, lo que significa que presentaban mayores tasas de falsas alarmas (tendían a responder siempre). Los análisis de regresión realizados para la edad y los índices obtenidos de la tarea CPT compleja (tasa de aciertos, tasa de falsas alarmas, d' y $\beta$ ) mostraron una relación no lineal en función de la edad para todos los criterios excepto en el caso de $\beta$. Esta relación cuadrática mostró que la atención sostenida aumenta de 6 a 9 años de forma rápida, se mantiene entre 9 y 11 , aumenta a los 12, y alcanza su máximo a los 13 años. Los resultados de este estudio mostraron que no había diferencias entre chicas y chicos en el CPT sencillo pero las chicas mostraban menor tasa de aciertos y menor d' que los chicos en el caso del CPT más complejo.

Kanaka, Matsuda, Tomimoto, Noda, Matsushima et al. (2008) también analizaron el desarrollo de la atención con un CPT simple. El estímulo diana (el número 9) aparecía el $20 \%$ de las veces. Evaluaron a 518 niñas japonesas de 5 a 12 años de edad. Los resultados obtenidos son congruentes con los de Lin, Hsiao y Chen, (1999): la inhibición, la desatención y la estabilidad del tiempo de procesamiento maduran en primer lugar. A partir de estos logros madurativos, aumenta la capacidad de discriminación y por último se reduce el tiempo de procesamiento.

A pesar de las diferencias existentes entre estudios (instrumentos, tamaño y representatividad de las muestras, rango de edad e índices) es interesante observar que sus resultados son bastante coincidentes: (1) el control ejecutivo de la actuación o inhibición mejora hasta los 6-7 y se estabiliza; (2) la atención sostenida y selectiva mejoran hasta los 10 y se estabilizan y vuelve a aumentar de los 12 a los 15 .

A la espera de disponer de una teoría unificada del desarrollo de la atención en un marco conceptual y teórico claro que incluya una descripción detallada de los tipos de atención y del modo de medir cada uno de ellos (Lehman et al., 2010) es necesario al menos: a) describir los distintos tipos de tareas y los tipos de atención que podemos medir a través del rendimiento en ellas; b) estudiar las diferencias entre los distintos tipos de variables de rendimiento (proporción de aciertos o velocidad); c) considerar el 
gradiente de complejidad de la tarea en relación a la edad de la muestra; d) considerar el gradiente de motivación (aburrimiento) que induce la tarea y d) calcular si, efectivamente, por las variables de rendimiento en una sola tarea, se pueden medir los tres diferentes tipos de atención sobre los que parece haber consenso actualmente.

Por lo tanto, el objetivo de este estudio es desarrollar dos tareas informatizadas, amigables y motivadoras para niños de 6 a 12 años, de breve administración, que permitan medir objetivamente los criterios que establece el DSM-IV-TR para el diagnóstico de TDAH. Para ello el primer paso es describir cada criterio de TDAH en operaciones de medida para, en un segundo momento, conocer cómo evolucionan estos índices y qué valores son propios de cada edad.

Los tres conceptos que según el DSM-IV-TR deben evaluarse para tomar las decisiones diagnósticas son: (1) Desatención; (2) Hiperactividad e (3) Impulsividad, teniendo en cuenta que la hiperactividad no se da en todos los casos.

El Manual DSM-IV-TR incluye, para cada uno de estos conceptos, un conjunto de descriptores de los que sólo vamos a considerar los referidos a los conceptos que se relacionan con la atención: 1) Desatención (mantenimiento y focalización de la atención) y 2) Impulsividad (control de la ejecución e inhibición de las respuestas automáticas).

\section{Las tareas de evaluación}

El grupo de investigación Psi+D de la UAM ha diseñado dos pruebas objetivas (test informatizados) de diferente grado de dificultad para medir la atención en niños, tratando de superar los problemas de las pruebas objetivas de discriminación visual de lápiz y papel (ausencia de registro automático de la ejecución del niño) y los problemas derivados de las pruebas del tipo actuación continua (CPT) en cuanto a su aplicación a niños (el parpadeo se confunde con desatención-distracción). Las dos tareas DiViSAUAM y TACI-UAM, se han desarrollado para ser administradas a través de ordenador (usando ratón o pantalla táctil) de forma que el programa registra secuencialmente el momento y tipo de acción de quien realiza la prueba. A partir de estos registros se obtienen diferentes índices que evalúan la atención y sus componentesa partir de la ejecución del niño. Cada uno de estos índices valora un aspecto diferente de la atención a partir del modo en que se realiza la tarea, del ajuste del niño a la demanda de la tarea y del nivel de rendimiento alcanzado.

Las pruebas fueron diseñadas como juegos para que el rendimiento en las mismas no estuviera influido por el aburrimiento, la falta de motivación o la pura discriminación perceptiva. Se desarrollaron con el objetivo de medir el desarrollo de la atención en niños de modo que permitan detectar el déficit de atención en niños con sospecha de TDAH. Este planteamiento exigió un extenso análisis conceptual para relacionar las variables de atención, los descriptores de TDAH y los índices de las pruebas diseñadas. Véase Anexo I para una descripción detallada.

En este estudio se describen con detalle las dos tareas desarrolladas y se analizan los diferentes índices obtenidos en cada una de las pruebas para cada grupo de edad (niños de 7 a 11 años).

\section{Método y procedimiento}

\section{Participantes}

En el estudio participaron 446 escolares, 23 de los cuales no fueron incluidos en los análisis de datos por hallarse en programas de adaptación curricular. Proceden de tres colegios de Madrid, dos de ellos públicos y uno subvencionado. De ellos 68 pertenecían a segundo curso (35 niñas y 33 niños), 112 a tercero (54 niñas y 58 niños), 79 a cuarto (48 niñas y 31 niños), 66 a quinto (27 niñas y 39 niños) y 98 a sexto (41 niñas y 57 niños). De la muestra finalmente analizada (423) 205 eran niños y 218 niñas.

\section{Instrumentos}

El Test de Discriminación Visual Simple de Árboles DiViSA-UAM es un test informatizado en el que el niño debe pulsar con el ratón (o con el dedo si se usa pantalla táctil) en los elementos (árboles) iguales al modelo. El árbol modelo varía de una pantalla a la siguiente. Consta de 8 pantallas, o ítems sucesi- 
vos. Cada pantalla se compone de una matriz de 12 x 12 en la que hay colocados 14 árboles iguales al modelo (10\% de la matriz) y 30 árboles distintos al mismo, mientras que el resto de las cuadrículas de la matriz están vacías (Figura 1). El árbol modelo varía de pantalla a pantalla. La primera pantalla es de familiarización y entrenamiento por lo que los resultados se obtienen a partir de la ejecución de los ítems 2 a 8 .

Cada pantalla tiene una duración máxima de 60 segundos y el niño puede pasar a la siguiente (pulsando Continuar) cuando considera que ha pulsado todos los elementos iguales al modelo. Al pulsar

Figura 1. Pantalla correspondiente a uno de los ítems del DiViSAUAM. A la derecha se presenta el árbol modelo



sobre los distintos árboles que componen cada pantalla estos desaparecen sólo si son iguales al árbol modelo, situado a la derecha de la pantalla.

Las instrucciones se presentan en dos pantallas previas a la tarea. En la primera pantalla junto al dibujo de un ítem se dice: "En este juego hay un tablero lleno de árboles. No todos los árboles son iguales. Si pulsas los árboles iguales al modelo que está a la derecha ganas puntos. Pulsa los árboles iguales al modelo en el menor tiempo posible." En la segunda dice: "Cuando estés listo pulsa COMENZAR y empezará la prueba. Se puede pasar de una a otra pantalla para revisar las instrucciones.

El programa registra completamente la ejecución del niño en cuanto a tipo de pulsación y momento en que la hace y de ello se extraen las siguientes varia- bles, para cada ítem: (1) tiempo de respuesta (TR), que es el tiempo entre pulsaciones, es decir el tiempo que transcurre hasta que pulsa sobre cada uno de los botones; (2) duración del ensayo, tiempo transcurrido desde la primera pulsación hasta que pulsa "Continuar" para pasar al siguiente ítem; (3) aciertos, número de árboles pulsados iguales al modelo; (4) errores de comisión, número de árboles pulsados no iguales al modelo; (5) errores de omisión, número de árboles iguales al modelo no pulsados y (6) orden en el que se pulsan los árboles que son iguales al modelo.

Sobre estas variables se construyeron los siguientes índices globales: (1) índice de atención global; (2) errores de comisión; (3) intervalo entre pulsaciones; (4) labilidad de la atención inter ensayos; (5) errores de omisión; (6) índice de organización y orden de la ejecución y (7) índice de distracción-precipitación.

El Test de Aprendizaje de Categorías - Inhibición. (TACI-UAM) es un test informatizado en el que el niño debe pulsar con el ratón (o con el dedo si se usa pantalla táctil) sobre las fichas para obtener puntos. Las fichas representan cuadrúpedos (9 puntos), vehículos (3 puntos), plantas/frutas (1 punto), y animales no cuadrúpedos (0 puntos). El niño desconoce a priori el valor de las fichas de cada categoría y debe ir asociando categoría a puntuación para lograr más puntos. En cada pantalla hay una matriz de $15 \times 15$ celdillas de las que 45 son cuadrúpedos (fichas con valor 9 puntos), 60 corresponden a otras figuras (21 son de 3 puntos; 20 son de 1 punto y 19 son de 0 puntos) y 120 son casillas en blanco. Las pantallas cambian de color y en las instrucciones se especifica que se pueden pulsar las fichas siempre que la pantalla no esté en amarillo. Cuando la pantalla cambia al amarillo, suena un tono agudo que avisa adicionalmente de dicho cambio. Al pulsar sobre la pantalla en amarillo se obtienen los puntos establecidos, pero como se advierte en las instrucciones, los puntos obtenidos estando la pantalla en amarillo se restan del total de los obtenidos en las pantallas de otros colores. El test consta de 8 ítems o problemas. El primero se considera de familiarización con la tarea y los resultados no se computan. El tiempo total para cada problema (pantalla) es de 43 segundos. En cada problema la pantalla cambia de color 7 veces (en tres 
Figura 2. Pantalla correspondiente a un Ítem del TACI-UAM en el que hay pulsados dos objetos en los que se ha obtenido 9 puntos en cada uno

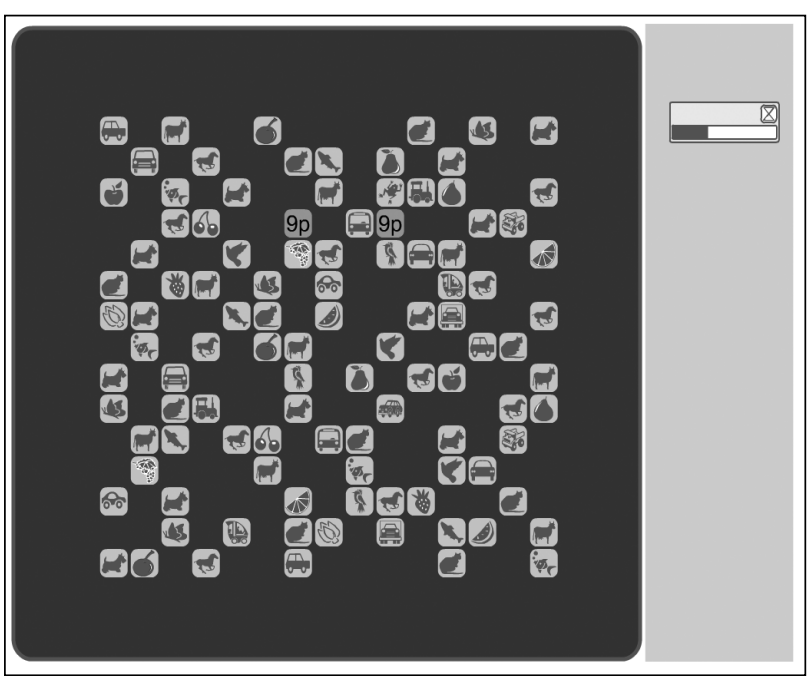

ocasiones a amarillo). Las figuras cambian de posición de un problema a otro. En la figura 2 se muestra un ítem en el que se han pulsado algunas fichas.

Las instrucciones se presentan en tres pantallas previas a la tarea. En la primera pantalla junto al dibujo de un ítem se dice: "En este juego hay un tablero con dibujos de animales y cosas. Hay caballos, coches, pájaros, cerezas y más cosas. ¡Mira el tablero! Si pulsas las fichas del tablero ganas puntos. Unas fichas tienen muchos puntos y otras tienen pocos, pero no sabemos qué fichas tienen más. En la segunda pantalla se dice: "Si pulsas sobre las fichas aparecen puntos debajo. Hay fichas que tienen 0 puntos y otras tienen 1,3 y hasta 9 puntos. Tienes poco tiempo así que pulsa primero aquellas fichas que tienen más puntos. Consigue el máximo número de puntos en cada tablero.” En la tercera pantalla se dice: "Cuidado, no pulses cuando la pantalla esté en amarillo. Si pulsas cuando esté en amarillo perderás los puntos". Después de cada ítem se informa a los niños de los puntos obtenidos en el mismo, teniendo en cuenta que cuando la pantalla está en amarillo los puntos obtenidos se restan. De esta manera el niño comprueba la correspondencia entre las instrucciones y la propia ejecución.

El programa registra completamente la ejecución del niño: tipo de pulsación y momento en que la hace y de ello se extraen las siguientes variables, para cada ítem o problema: (1) tiempo de respuesta (TR); (2) aciertos; (3) errores de comisión; (4) errores de omisión; (5) secuencia de pulsaciones; (6), número de fichas pulsadas cuando la pantalla está en amarillo; y (7) total de pulsaciones.

Sobre estas variables se construyeron los siguientes índices globales: 1) índice de atención global; 2) errores de comisión; 3) intervalo entre pulsaciones; 4) errores de omisión; (5) índice de organización y orden de la ejecución; (6) índice de distracciónprecipitación y (7) control de la actuación (inhibición).

En el test TACI-UAM, a diferencia del DiViSAUAM, no se toma en consideración la labilidad atencional, puesto que la varianza intra-ítem de cada niño en el índice de atención global, se debe al progresivo aprendizaje de la tarea. Sin embargo, se puede medir la dificultad para esperar dado que el TACI exige que en el tiempo en que la pantalla está en amarillo no se pulse sobre las fichas. Ninguna de las dos pruebas puede medir, en su configuración actual, la distracción ante lo irrelevante o el desagrado y aversión ante el esfuerzo. Este aspecto queda pendiente de desarrollar en futuras versiones.

\section{Procedimiento}

Para evaluar a los escolares se utilizaron las aulas informáticas de los colegios. Los niños fueron evaluados colectivamente en grupos de 15. En primer lugar se administró siempre el DiViSA-UAM y en segundo lugar el TACI-UAM.

\section{Análisis de Datos}

En el análisis de datos se utilizó el paquete estadístico SPSS-17. En primer lugar se calcularon, a partir de las variables por ítem de cada niño, los índices descritos para cada test. En segundo lugar, se analizaron las distribuciones de cada variable por curso y se calculó la fiabilidad de las puntuaciones de los índices obtenidos; se obtuvieron los estadísticos descriptivos para cada curso y se realizaron análisis múltiples de varianza para cada test para analizar si el perfil de rendimiento en las tareas varía en función de la edad. Tras esta comprobación, se realizaron análisis de varianza para cada variable para determinar cuáles de ellas tienen más peso en la 
diferenciación por cursos. En tercer lugar se ha llevado a cabo un análisis de correlaciones entre las variables obtenidas con cada test. En el TACI, además de los índices de atención previamente descritos se ha calculado el Índice de aprendizaje (proporción de pulsaciones sobre figuras de 9 puntos /total de figuras pulsadas), obtenible del registro general de datos del TACI-UAM. Las correlaciones entre los índices de atención del TACI y el índice de aprendizaje, mostrarán en qué medida la falta de atención está asociada a la dificultad para aprender. En cuarto lugar se calcularon las correlaciones entre los índices de las dos pruebas para analizar su convergencia.

\section{Resultados}

\section{Fiabilidad de las variables}

Para cada índice de atención de cada uno de los tamaño medio y coherentes según su signo (Por ejemplo, a mayor puntuación en atención, menor puntuación en errores de comisión, de omisión y menor intervalo entre pulsaciones). Las correlaciones entre Organización y Distracción- Precipitación con el resto de variables, son cercanas a cero aunque algunas son significativas debidas al elevado tamaño de la muestra. En el caso de la variable Organización esto indica que en estas edades el índice de Organización del DiViSA-UAM refleja diferencias de estilo, no de edad. En el caso del índice Distracción-Precipitación, las bajas correlaciones con las demás variables son fruto de su esencia bipolar (puede haber niños con la misma puntuación en el Índice de Atención Global que muestren puntuaciones opuestas en el Índice de DistracciónPrecipitación de forma que en unos casos la poca atención cursa con distracción-retirada de atención -puntuaciones positivas altas- mientras que en otros casos la poca atención cursa con precipitación -pun-

Tabla 1. Fiabilidad de los índices de atención e impulsividad del DiViSA-UAMy Correlaciones entre sus variables

\begin{tabular}{|c|c|c|c|c|c|c|c|}
\hline & Atención & Comisiones & $\begin{array}{c}\text { Intervalo entre } \\
\text { pulsaciones }\end{array}$ & Omisiones & Labilidad & Organización & $\begin{array}{l}\text { Distracción } \\
\text { Precipitación }\end{array}$ \\
\hline Atención Global & - & & & & & & \\
\hline Comisiones & $-.337 * *$ & - & & & & & \\
\hline Intervalo entre pulsaciones & $-.666 * *$ & $.112 *$ & - & & & & \\
\hline Omisiones & $-.239 * *$ & $.253 * *$ & $.485 * *$ & - & & & \\
\hline \multicolumn{8}{|l|}{ Labilidad de atención } \\
\hline Inter ensayos (SD) & $.196 * *$ & $.412 * *$ & -.077 & $.223 * *$ & - & & \\
\hline Organización & -.063 & -.041 & -.008 & $-.173 * *$ & -.066 & & - \\
\hline Distracción-Precipitación & .043 & $-.272 * *$ & .015 & .035 & $-.170 * *$ & -.007 & - \\
\hline Fiabilidad $(\alpha)$ & .95 & .86 & $\alpha .94$ & $\alpha .77$ & - & - & - \\
\hline
\end{tabular}

Nota. $* p<.05 ; * * . p<.01$

test se calculó el alfa de Cronbach. En la tabla 1 se puede observar la fiabilidad de los índices referidos a atención en el DiViSA-UAM. Los índices Atención global, Errores por comisión, Errores por omisión y Velocidad, medida por el intervalo entre pulsaciones, son altos. Sin embargo los índices de Organización y de Labilidad atencional incluidos en el concepto de Desatención en el DSM-IV-TR y el índice de Distracción-Precipitación incluido en el de Impulsividad, no muestran consistencia en estos rangos de edad. Las correlaciones entre las variables de atención del DiViSA-UAM son, en su mayoría, de tuaciones negativas altas-). Por último, el intervalo entre pulsaciones (velocidad) es independiente de la labilidad de la atención inter-ensayos.

En la Tabla 2 se incluye la fiabilidad de los índices referidos a atención en el TACI-UAM y las correlaciones entre sus variables. La fiabilidad de todos los índices proporcionados por el TACI-UAM es elevada siendo los valores obtenidos superiores a 0.90. Las correlaciones del índice global de Atención con los Errores por Omisión y Comisión son también altas mientras que la relación con el intervalo entre pulsaciones en esta prueba es menor, 
Tabla 2. Fiabilidad de los índices de atención, impulsividad y rendimiento del TACI-UAM y Correlaciones entre sus variables

\begin{tabular}{|c|c|c|c|c|c|c|c|}
\hline & Atención & Comisiones & $\begin{array}{l}\text { Intervalo entre } \\
\text { pulsaciones }\end{array}$ & Omisiones & Organización & $\begin{array}{l}\text { Deshinibición } \\
\text { actuación }\end{array}$ & Aprendizaje \\
\hline Atención & - & & & & & & \\
\hline Comisiones & $-0.804 * *$ & - & & & & & \\
\hline Intervalo entre pulsaciones & $0.249 * *$ & $-0.690 * *$ & - & & & & \\
\hline Omisiones & $-0.746 * *$ & $0.204 * *$ & $0.363 * *$ & - & & & \\
\hline Organización & $0.927 * *$ & $-0.638 * *$ & 0.062 & $-0.812 * *$ & - & & \\
\hline Desinhibición actuación & $-0.170 * *$ & $0.275^{* *}$ & $-0.231 * *$ & -0.028 & -0.086 & - & \\
\hline Aprendizaje & $0.937 * *$ & $-0.892 * *$ & $0.516^{* *}$ & $-0.543 * *$ & $0.819 * *$ & $-0.203 * *$ & - \\
\hline Fiabilidad $(\alpha)$ & .96 & .96 & .93 & .95 & .89 & .93 & .95 \\
\hline
\end{tabular}

Nota. $* p<.05 ; * * . p<.01$.

debido probablemente a que los ensayos tienen un tiempo fijo. En esta prueba el índice de atención Global también correlaciona alto con Organización. $\mathrm{El}$ intervalo entre pulsaciones se asocia, igual que en el DiViSA-UAM, con los errores de omisión. La Desinhibición ante la actuación muestra correlaciones con las variables esperadas (positiva con los errores de comisión y negativa con el intervalo entre puntuaciones) aunque bajas. El nivel de aprendizaje, medido por la proporción de pulsaciones sobre las fichas de 9 puntos, correlaciona coherentemente con todas las variables mostrando, como se preveía, que la falta de atención y de inhibición de la actuación dificulta el aprendizaje.

\section{Análisis de Diferencias entre Cursos (influencia de la edad)}

Para estudiar el desarrollo de la atención mediante estos test, se realizaron dos análisis múltiples de varianza (MANOVA), uno para cada test, con el curso y el sexo como variables independientes y como variables dependientes los índices de cada uno de los test estudiados. Ni el sexo ni la interacción produjeron efectos estadísticamente significativos. Sólo el curso se asocia con un perfil diferente en cada test (Raíz Mayor de Roy $(6,416)=.65 ; p<$ $.001 ;$ eta $^{2}=.39$ para el DiViSA-UAM y Raíz Mayor de Roy $(6,416)=.88 ; p<.001 ;$ eta $^{2}=.47$ para el TACI-UAM). En las Tablas 3 y 4 se incluyen los estadísticos descriptivos por cursos así como los estadísticos de contraste y la contribución de cada variable a la explicación de las diferencias de perfil entre cursos $\left(\mathrm{eta}^{2}\right)$.

Los datos de la Tabla 3 referidos al DiViSA-UAM muestran que la variable atención global aumenta de forma suave pero constante a lo largo de los cursos. Los Errores por comisión decrecen bruscamente en tercer curso, mientras que los errores por omisión, van decreciendo hasta $5^{\circ}$ curso. El intervalo entre pulsaciones decrece de segundo a tercero y se mantiene para volver a decrecer en sexto. La Labilidad de la Atención entre los ensayos, la Organización y

Tabla 3. Estadísticos descriptivos por curso. de las variables obtenidas a partir del DiViSA-UAM

\begin{tabular}{|c|c|c|c|c|c|c|c|c|c|c|c|c|}
\hline \multirow[b]{2}{*}{ DiViSA-UAM. } & \multicolumn{2}{|c|}{$2^{\circ}$ Curso $n=68$} & \multicolumn{2}{|c|}{$3^{\circ}$ Curso $n=112$} & \multicolumn{2}{|c|}{$4^{\circ}$ Curso $n=79$} & \multicolumn{2}{|c|}{$5^{\circ}$ Curso $n=66$} & \multicolumn{2}{|c|}{$6^{\circ}$ Curso $n=98$} & \multirow[b]{2}{*}{$\mathrm{F}$} & \multirow[b]{2}{*}{ eta } \\
\hline & M & SD & M & SD & M & $\mathrm{SD}$ & M & $\mathrm{SD}$ & M & $\mathrm{SD}$ & & \\
\hline Atención & 0.44 & 0.12 & 0.55 & 0.11 & 0.57 & 0.15 & 0.63 & 0.15 & 0.76 & 0.17 & $50.17 * * *$ & .35 \\
\hline Comisiones & 3 & 6.38 & 1.29 & 2.48 & 1.47 & 4.62 & 1.45 & 2.78 & 0.61 & 1.86 & $3.75^{* *}$ & .04 \\
\hline Intervalo entre pulsaciones & 2.22 & 0.85 & 1.74 & 0.33 & 1.74 & 0.52 & 1.65 & 0.87 & 1.40 & 0.91 & $12.24 * * *$ & .11 \\
\hline Labilidad inter ensayos & 0.12 & 0.05 & 0.11 & 0.04 & 0.13 & 0.06 & 0.14 & 0.06 & 0.15 & 0.05 & $5.42 * * *$ & .05 \\
\hline Omisiones & 4.19 & 4.15 & 2.98 & 3.26 & 2.46 & 3.19 & 1.56 & 3.55 & 1.84 & 2.64 & $6.67 * * *$ & .07 \\
\hline Organización & 17.29 & 3.50 & 18.12 & 3.89 & 17.36 & 3.22 & 17.59 & 3.87 & 17.93 & 4.37 & 0.71 & .01 \\
\hline Distracción-Precipitación & 0.76 & 1.30 & 0.68 & 1.15 & 0.68 & 1.10 & 0.44 & 1.45 & 0.71 & 0.80 & 0.74 & .01 \\
\hline
\end{tabular}

Nota: $* * p<.01 ; * * * p<.001$. 
Tabla 4. Estadísticos descriptivos por cursos, de las variables obtenidas a partir del TACI-UAM

\begin{tabular}{|c|c|c|c|c|c|c|c|c|c|c|c|c|}
\hline \multirow[b]{2}{*}{ TACI-UAM. } & \multicolumn{2}{|c|}{$2^{\circ}$ Curso $n=68$} & \multicolumn{2}{|c|}{$3^{\circ}$ Curso $n=112$} & \multicolumn{2}{|c|}{$4^{\circ}$ Curso $n=79$} & \multicolumn{2}{|c|}{$5^{\circ}$ Curso $n=66$} & \multicolumn{2}{|c|}{$6^{\circ}$ Curso $n=98$} & \multirow[b]{2}{*}{$\mathrm{F}$} & \multirow[b]{2}{*}{ eta $^{2}$} \\
\hline & M & $\mathrm{SD}$ & M & $\mathrm{SD}$ & M & $\mathrm{SD}$ & $\mathrm{M}$ & $\mathrm{SD}$ & M & $\mathrm{SD}$ & & \\
\hline Atención & 2.74 & 6.32 & 3.93 & 6.21 & 6.99 & 8.08 & 11.18 & 10.72 & 10.96 & 10.79 & $16.89 * * *$ & .14 \\
\hline Comisiones & 10.46 & 4.51 & 11.93 & 5.23 & 9.86 & 6.43 & 9.99 & 7.67 & 11.69 & 7.02 & 2.14 & .02 \\
\hline Intervalo entre pulsaciones & 1.59 & 0.53 & 1.36 & 0.57 & 1.53 & 0.70 & 1.33 & 0.68 & 1.11 & 0.47 & $8.93 * *$ & .08 \\
\hline Omisiones & 31.77 & 4.03 & 29.14 & 3.35 & 28.15 & 4.47 & 23.83 & 4.93 & 22.35 & 5.34 & $62.67 * * *$ & .38 \\
\hline Organización & 7.03 & 6.42 & 8.17 & 5.78 & 11.04 & 7.73 & 16.44 & 9.46 & 15.90 & 9.91 & $24.16^{* * *}$ & .19 \\
\hline Inhibición actuación & 25.97 & 29.86 & 26.92 & 29.00 & 22.52 & 22.82 & 18.42 & 9.10 & 20.56 & 13.68 & 2.07 & .02 \\
\hline Aprendizaje & 0.51 & 0.16 & 0.53 & 0.16 & 0.61 & 0.20 & 0.67 & 0.22 & 0.63 & 0.20 & $9.95^{* * *}$ & .09 \\
\hline
\end{tabular}

Nota: $* * p<.01 ; * * * p<.001$.

la Distracción - Precipitación se mantienen relativamente estables a lo largo de los cursos en esta prueba.

Por lo que se refiere al TACI-UAM, los datos de la comparación entre cursos (Tabla 4) muestran que el nivel de atención global crece paulatinamente a lo largo de los cursos. Los errores por omisión decrecen suave y lentamente a lo largo de los cursos, mientras que los errores por comisión se mantienen relativamente estables. La Organización en esta prueba juega un papel importante, creciendo hasta $5^{\circ}$ curso, momento a partir del cual se mantiene.

Por último en la Tabla 5 se muestran las correlaciones entre los índices de cada una de las pruebas, sin considerar Labilidad de la Atención porque en el TACI -UAM en esta variable, que refleja la varianza entre ensayos en el índice de atención global, una puntuación alta es señal de aprendizaje y no de variabilidad en el despliegue de la atención, como ocurre en el DiViSA-UAM, por lo que no se ha calculado. La variable Distracción- Precipitación no se ha podido calcular con los datos registrados en la versión actual del TACI-UAM y por ello tampoco está incluida. Los datos muestran correlaciones significativas del orden de $r=.37$ entre los índices de atención global y los índices de velocidad entre ambas pruebas.

\section{Discusión}

En este estudio se han descrito los resultados obtenidos con dos pruebas nuevas desarrolladas para medir atención (sostenida y selectiva, fundamentalmente) en niños de 6 a 12 años de edad. Ambas pruebas se han administrado a 423 escolares de $2^{\circ}$ a $6^{\circ}$ curso de primaria, para analizar el patrón evolutivo de las diversas medidas que proporcionan.

El test DiViSA es un test basado en la rapidez de discriminación visual mientras que el TACI es un test basado en la discriminación de categorías de fichas. En el DiViSA, el niño se toma el tiempo que considera necesario para hacer la tarea y, por tanto,

Tabla 5. Correlaciones entre los índices de atención de las dos pruebas

\begin{tabular}{llllll}
\hline & Atención & Comisiones & $\begin{array}{c}\text { Intervalo } \\
\text { pulsaciones }\end{array}$ & Omisiones & $\begin{array}{c}\text { Organización } \\
\text { Distracción- } \\
\text { Precipitación }\end{array}$ \\
\hline DiViSA- UAM & $\begin{array}{l}\text { Atención Global } \\
\text { Comisiones } \\
\text { Intervalo entre } \\
\text { Pulsa. Velocidad } \\
\text { Omisiones } \\
\text { Organización }\end{array}$ & $.376^{* *}$ & .045 & & \\
& & & $.368^{* *}$ & $.191^{* *}$ & .008 \\
\hline
\end{tabular}

$* * p<.01$ 
la medida de la atención se asienta en el tiempo que el niño invierte en cada ensayo. La tarea es de discriminación visual simple y, resolverla, no requiere de algún tipo de capacidad compleja ligada a inteligencia. En el TACI la tarea requiere atención asociada al premio que se obtiene según la categoría a la que pertenece un objeto del conjunto y exige la identificación de las figuras pertenecientes a dicha categoría (en este caso cuadrúpedos). Esta es una tarea más compleja que las habituales de discriminación y búsqueda visual, no por la dificultad de la discriminación perceptiva a realizar, sino porque la discriminación es conceptual y hay que descubrirla a partir de la actuación en la tarea.

\section{Características diferenciales del DiViSA-UAM y el TACI-UAM en relación a otras pruebas de atención}

Las 2 pruebas desarrolladas (DiViSA-UAM y TACI-UAM) por el grupo PSi+D de la UAM y configuradas para medir la atención en niños de 6 a 12 años presentan, a nuestro modo de ver, diversas ventajas en la evaluación de la atención en niños, en comparación con otras pruebas disponibles.

En primer lugar, el formato de administración mediante ordenador, frente al de lápiz y papel, permite registrar la ejecución individual de cada niño tanto en las variables de tiempo como en las de logro. El soporte informatizado también permite la evaluación colectiva, bien instalándolo en varios ordenadores u On-line mediante un servidor WEB, además, el programa puede ser ejecutado tanto en ordenadores con ratón como en ordenadores con pantalla táctil. Como toda administración informatizada, libera al evaluador de tener que anotar la ejecución del niño pudiendo dedicarse a observar otros aspectos cualitativos de su comportamiento al realizar el test.

En segundo lugar respecto al contenido, los test de discriminación visual que se emplean actualmente en España (D2, Brickenkamp, 2002; Test de Caras, Thurstone y Yela, 1985 y Escala Magallanes de Atención Visual, -EMAV- García y Magaz, 2000), miden velocidad de procesamiento perceptivo y consisten en una gran matriz de estímulos, muy similares entre sí, en donde el niño debe encontrar y marcar aquellos que se corresponden con el estímulo modelo, lo más rápidamente que pueda, durante un tiempo determinado. Tanto el DiViSA-UAM como el TACIUAM, requieren menos capacidad discriminativa y un menor nivel de concentración, por lo que permiten evaluar niños desde los 6 años sin que el rendimiento en los test pueda confundirse con las capacidades intelectuales o visuales requeridas por la tarea.

En tercer lugar, frente a los test de actuación continua, (CPT, Continuous Performance Test) como el Test de Atención Sostenida para Niños (CSAT, Servera y Llabrés, 2004) cuya administración también es informatizada, el DiVisa-UAM y el TACIUAM no presentan los estímulos de forma secuencial (con una latencia entre uno y otro que puede variar desde unos milisegundos hasta varios segundos), sino que cada ítem incluye diversos elementos (14 árboles iguales al modelo en el DiViSA y 45 elementos de la categoría que recibe 9 puntos en el TACI) esparcidos por un campo de información que contiene otros elementos (30 árboles no iguales al modelo en el DiViSA y 60 fichas de las restantes categorías en el TACI). Este formato de tarea es más semejante al de las actividades escolares, lo que aumenta la validez ecológica de las dos pruebas desarrolladas. La presentación serial de información impide valorar cómo se enfrenta el niño a un campo de información con más de un estímulo. La presentación simultánea de la información relevante, característica del DiViSA y el TACI, hace que los errores de omisión se deban a una falta de atención durante la realización de la tarea o a una ejecución rápida o descuidada, y no a que el niño parpadee o desvíe la mirada de la pantalla repetidamente, como ocurre en los test de actuación continua. Frente al uso de letras o números en los CPT, el DiViSA-UAM y el TACI emplean figuras (árboles en el DiVisa, cuadrúpedos, coches, plantas y animales no cuadrúpedos en el TACI), lo que evita los falsos positivos que se suelen producir con los niños con problemas de lectoescritura y aprendizaje (McGee, Clark, Symons, 2000). El hecho de que los árboles iguales al modelo desaparezcan al pulsarlos, en el DiViSA, o que aparezcan los puntos obtenidos al pulsar la ficha en el TACI, hace que los niños perciban las pruebas como un juego, lo cual facilita que su motivación se mantenga alta durante la ejecución de la prueba. 


\section{Ajuste a los descriptores del DSM-IV}

En el diseño del DiViSA y del TACI se tuvieron en cuenta los criterios DSM-IV-TR (2002) para que ambas pruebas permitieran medir esos criterios en escolares y así conocer los patrones evolutivos de cada medida. Conocidos los patrones evolutivos de cada uno de los índices, estos dos test podrán utilizarse para valorar objetivamente la desviación a su grupo de edad de un niño con dificultades de atención y/o sospecha de TDAH.

Tanto el DiViSA como el TACI incluyen medidas de tiempo y medidas de logro. Esto es relevante porque la precisión, los errores -y su tipo- y la velocidad, son los indicadores usualmente utilizados para el diagnóstico de TDAH (Barkley, 1991; Conners, Epstein, Angold y Klaric, 2003; Epstein, Erkanli, Conners, Klaric, Costello, y Angold, 2003; Willcutt, Pennington, Boada, Ogline, Tunick, et al., 2001; Willcutt, Pennington, Olson, Chabildas, y Hulslander, 2005). A partir de las medidas de tiempo y logro registradas en cada ítem, se han obtenido los 8 índices de atención analizados, 6 de los cuales son comunes en ambas pruebas.

No obstante el diseño de dos pruebas nuevas requiere comprobaciones adicionales que deben realizarse en los siguientes estudios (por ejemplo rangos de edad en los que son de mayor utilidad cada una de las pruebas).

\section{Características psicométricas y consistencia de los indicadores}

Los coeficientes de fiabilidad obtenidos para los índices calculados, son altos $(\alpha>$.90) exceptuando los errores, de comisión y de omisión, del DiViSA cuyos valores son .86 y .77 . Los valores de consistencia interna obtenidos indican que ambas pruebas captan de forma sistemática a través de los ítems, la forma en que los niños despliegan la atención, focalizan y discriminan al enfrentarse a un campo de información complejo. El hecho de que sean sólo los errores del DiViSA los que presentan menor consistencia se debe a la facilidad de la prueba que hace que haya pocos errores en todos los cursos evaluados.
Las correlaciones entre los índices de atención del DiViSA-UAM aunque pequeñas $(\mathrm{r}=.25 \mathrm{a} \mathrm{r}=.47)$, son estadísticamente significativas y coherentes según su signo, tal y como se ha descrito en el epígrafe de resultados. La magnitud de estas correlaciones, como indicadores de validez convergente, es semejante a la que muestran muchos test de capacidad en niños (véase por ejemplo la batería DAT-5 de Bennet, Seashore y Wessman, 1990 o el PMA del Departamento I+D de TEA, 2008). Así, a mayor puntuación en el índice de atención global, menor puntuación en errores de comisión, de omisión y menor intervalo entre pulsaciones (mayor rapidez en la ejecución). No obstante, debe resaltarse que no se esperaban correlaciones altas por cuanto se parte del supuesto de que a una misma puntuación de atención global pueden corresponder número de errores de omisión y comisión distintos, diferentes intervalos entre puntuaciones, distracción o precipitación.

Como han señalado otros investigadores, para valorar las deficiencias de atención deben tenerse en cuenta tanto los errores como los tiempos (Castellanos y Tannock, 2002; Epstein et al., 2003) y las diversas combinaciones que pueden darse entre ellos. Por ello algunos autores plantean medir la precipitación (medida de tiempo) por los errores (medida de precisión) que se cometen en una prueba como CEFT, (López, Delgado, Pérez, Serrano, Alberola, Andrés, Sacristan y Camina, 2010). En el DiViSA, es interesante señalar que las variables Labilidad Inter-ensayos e Intervalo entre pulsaciones son independientes entre sí. La Labilidad inter-ensayos se relaciona con los errores de comisión, mientras que el intervalo entre puntuaciones se relaciona con las omisiones.

En el TACI las correlaciones del índice global de Atención con los Errores por Omisión y Comisión son altas (- $.80 \mathrm{y}-.75$, respectivamente) mientras que la relación con el intervalo entre pulsaciones es menor (-.25), debido probablemente a que los ensayos tienen un tiempo fijo. En esta prueba el índice de atención Global también correlaciona alto con Organización (.93) mostrando que el rendimiento en esta prueba sí está asociado a una ejecución organizada. La Desinhibición en la actuación muestra correlaciones con las variables esperadas (.28 con los errores de comisión y -.23 con el intervalo entre puntuaciones) lo que refleja que la falta de control 
sobre la actuación (o dificultad para esperar cuando la pantalla está en amarillo) lleva asociadas pulsaciones rápidas pero inexactas.

El TACI incluye además una medida del nivel de aprendizaje, aunque no se trata de un índice de atención, que muestra correlaciones altas $(>.50)$ con los demás índices excepto con el de Desinhibición. Este dato, aunque requiere ser replicado, indica que la desinhibición no lleva necesariamente asociada falta de aprendizaje mientras que las variables relacionadas con la desatención, sí.

En lo que respecta a las características psicométricas de las pruebas, en los próximos trabajos será necesario estudiar la estabilidad de las medidas, así como la convergencia con otras pruebas utilizadas para medir la atención en niños.

\section{Evolución de la atención en la edad escolar}

Los resultados obtenidos han mostrado ausencia de influencia del sexo en la ejecución de ambas pruebas tal y como obtuvieron Klenberg et al. (2001) con niños de 3 a 12 años y test de papel y lápiz y Lin et al. (1999) con niños de 6 a 15 años de edad y un CPT.

Al analizar el patrón evolutivo (por cursos) en ambas pruebas los resultados obtenidos muestran que: (a) la variable atención global aumenta de forma suave pero constante a lo largo de los cursos; (b) a partir de $3^{\circ}$ apenas se cometen errores de comisión si la tarea es de discriminación visual simple (DiViSA) mientras que decrecen paulatinamente de $2^{\circ}$ a $6^{\circ}$ si la tarea es más compleja (discriminación visual conceptual, TACI); (c) los errores por omisión decrecen paulatinamente de $2^{\circ}$ a $6^{\circ}$; (d) el intervalo entre pulsaciones decrece paulatinamente si la tarea es de discriminación visual simple, lo que muestra la menor velocidad de los más pequeños; sin embargo, si la tarea es más compleja, el intervalo entre pulsaciones no muestra un patrón evolutivo claro lo que indica que no depende tanto de la maduración; (e) la organización de la ejecución se incrementa hasta $5^{\circ}$ curso y se mantiene cuando la tarea es más compleja (TACI) mientras que no muestra ningún patrón evolutivo en la tarea más simple (DiViSA).

Estos resultados convergen y completan los encontrados por Klenberg et al. (2001), por Lin et al., (1999) y por Kanaka et al. (2008). Convergen porque muestran diferencias sistemáticas por curso en los índices globales y los complementan porque muestran que la continuidad o discontinuidad del patrón de desarrollo de la atención depende de las características de las tareas: en las tareas de discriminación visual simple (DiViSA) el patrón evolutivo del índice global de atención, de los errores y de la velocidad, muestra continuidad en la mejora (como en los resultados obtenidos con el CPT) mientras que en las tareas complejas (TACI) el patrón evolutivo es continuo en el índice de atención global y en los errores pero no en la velocidad. También los complementan porque rescatan las diferencias cualitativas (como el indicador $\beta$-criterio de respuesta- en los CPT) a través de diversos índices: labilidad atencional, organización y distracción-precipitación en el DiViSA y desinhibición en el TACI.

Estos resultados evolutivos se han obtenido en comparaciones transversales como en los estudios de Klenberg et al., (2001), Lehman et al. (2010), Lin et al. (1999) y Kanaka et al. (2008) con resultados semejantes, sin embargo, en futuros trabajos sobre el desarrollo de la atención, resultará imprescindible realizar estudios longitudinales que den cuenta de la evolución de la atención en cada niño, a partir de los diferentes estados iniciales.

\section{Utilización de las pruebas desarrolladas para el diagnóstico de TDAH}

El Trastorno por Déficit de Atención con o sin Hiperactividad (TDAH) es, en la actualidad, el problema más diagnosticado durante la infancia, de ahí el creciente interés en desarrollar métodos de evaluación, fiables, válidos y, sobre todo, eficaces en tiempo y dinero. Lo padecen entre un 3-5\% de niños menores de 10 años, siendo la prevalencia mayor en los varones (APA, 2000; Gomez, Harvey, Quick, Scharer, y Harris, 1999).

La atención, que habitualmente se entiende como un mecanismo regulatorio útil para percibir, en función de las demandas de contexto y los recursos de los que dispone el individuo, en ocasiones se ve como un sistema con un sesgo estable relativo al 
déficit o al exceso de atención que produce un menor rendimiento. En el caso de no percibir los elementos relevantes, el niño no discrimina y, cuando es el caso, no responde (error de omisión) o responde tarde (lentitud). En el caso contrario, en el que todos los elementos son relevantes, el individuo tampoco discrimina, responde tarde (lento) y comete errores de todo tipo (por omisión y por comisión). En resumen, y ésta es la paradoja, los sesgos del sistema regulatorio con independencia de su sentido, conllevan un bajo nivel de rendimiento e inducen errores y lentitud en la respuesta de los niños. Sin embargo, resulta evidente que la intervención que permitirá un mejor rendimiento dependerá del tipo de sesgo del mecanismo de atención del individuo. En unos casos será necesario presentar la tarea con estímulos más llamativos, duraderos e intensos, para que lleguen a ser relevantes e induzcan en el niño un mayor nivel global de activación y, en otros, será necesario reducir el nivel del ruido del contexto, para que contraste con la normal relevancia de los estímulos clave de la tarea $\mathrm{y}$, al mismo tiempo, reduzca el nivel global de activación del niño.

Varios estudios coinciden en señalar que el desarrollo de la atención se organiza a partir de la maduración del sistema de alerta, sobre el que se desarrolla el sistema de vigilancia que posibilita la focalización y selección y sobre los que, por último, maduran las funciones ejecutivas (Posner y Petersen, 1990; Posner y Raichle, 1994 y Santostefano 1978, 1985 y 1998). De este planteamiento se han derivado dos alternativas para la explicación del TDAH. La primera, pretende dar cuenta de la lentitud que se observa en algunos casos como un tipo específico de déficit de motivación cuya dificultad estriba en la incapacidad para detectar los elementos relevantes y en ocasiones poco sobresalientes de una tarea. La segunda, pretende dar cuenta de los errores que se producen, bien por distracción, olvido, desorganización, bajo nivel de motivación general o, alternativamente, dar cuenta de los errores que se producen por una actuación impulsiva -desinhibida- y descontrolada que se manifiesta en la precipitación.

Con estos modelos en mente, disponer de pruebas estandarizadas que permitan evaluar cada uno de estos aspectos supondría una ayuda para los profesionales en el proceso diagnóstico de TDAH. Los datos de este estudio muestran que el DiViSA y el TACI pueden servir a este propósito.

\section{Extended Summary}

This study analyses the developmental pattern of attention for Spanish school children ranging from 7 to 11 years old. Two new tests have been elaborated to test attention, both of them computerized. These tests have been elaborated by the Psi+D research group from the Universidad Autónoma of Madrid. The paper includes the descriptive statistics classified according to school grade.

As a starting point, the criteria for ADHD are briefly reviewed in terms of attention components: sustained attention (maintaining attention in simple and repetitive tasks), selective attention (focusing attention on relevant stimuli while discarding irrelevant ones), and executive control (being able to inhibit response to the stimuli in an automatic and non reflexive way).

Papers from Rueda, Fan, McCandliss, Halparin, Gruber, Pappert and Posner (2004); Klenberg, Korkman and Lahti-Nuutila (2001); and Lehman,
Naglieri and Aquilino (2010) about the development of attention are reviewed. This leads to the following conclusions:

1. Achievement in attention tasks progressively increases throughout the studied age range.

2 . The three attention components develop consecutively. First, motor inhibition and impulse control mature. Afterwards, sustained and selective attention mature.

3. Accuracy and speediness, the variables measuring achievement in attention tasks, are two different elements in the task solving process, which develop along different paths during childhood.

Lin, Hsiao and Chen, (1999) and Kanaka, Matsuda, Tomimoto, Noda, Matsushima et al. (2008) also carried out studies about the development of attention, but using the Continuous Performance Test (CPT). Results obtained from these stud- 
ies are congruent with the above-mentioned conclusions: motor inhibition, inattention and stability of invested time to solve the task mature first; later discrimination ability (selective attention) improves, and, finally, processing time reduces.

To obtain more precise data about the development of attention, it is necessary to describe concisely the different types of tasks used to measure the level of attention and the components of attention being measured through the achievement in those tasks. It is also necessary to study the differences between the two achievement variables (accuracy versus speediness of response). Last, but not least, when studying the development of attention it is crucial to take into account the task complexity and the motivation this task arises in children.

With previous studies as background, the Psi+D research group designed two objective tasks, which differ in difficulty, to measure attention in children. DiViSA-UAM is a test of simple visual discrimination and TACI-UAM is a test to measure category discrimination of an array of figures. Categories have different rewards associated to them and performance should be inhibited when the color of the screen turns yellow. Both tests were designed to overcome the problems that have arisen with paper and pencil (in terms of the discrimination ability required to solve them) and CPT tasks when administered to children. Tasks were developed to be similar to a computer game. Administration takes no longer than 8 minutes and the program records, sequentially, each mouse action and time moment.

\section{Method}

\section{Participants}

In the study, 423 school children were evaluated (51\% were boys) from three different schools in Madrid.

\section{Instruments}

The Test de Discriminación Visual Simple de Árboles DiViSA-UAM (Simple Visual Discrimina- tion of Trees Test) is a computerized test in which the child should select (by pressing with mouse or finger) the trees equal to the target one (Figure 1). Target tree differs from item to item. The test consists of 8 items (screens). Each item consists of a $12 \times 12$ matrix where 14 trees identical to the target $(10 \%)$ plus 30 trees different from the target are located. Resting cells are empty. The first screen serves as an example to familiarize the child with the task. To go to the next item, the child must press the "following" button when he/she considers that all trees have been selected. When a tree identical to the target tree is selected, it disappears.

The Test de Aprendizaje de Categorías Implícitas -TACI-UAM- (Implicit Categories Learning Test) is a computerized test used to learn to discriminate amongst objects. The child must press over each figure to obtain points (using a mouse or a finger). When pressing a figure, it turns over and shows the obtained points (Figure 2). Figures with quadruped animals give 9 points, those with vehicles give 3 points, figures with plants or fruits give 1 point and figures with non-quadruped animals give 0 points. When starting the task, the child doesn't know how many points can be obtained by pressing each type of figure; thus the child should associate each category to the obtained points and therefore press those figures which give more points. The screen changes its color several times during each item. Instructions specify that the child can press figures with every screen color except yellow. Pressing a figure when the screen is yellow means that, the points of that figure will be subtracted from the amount of obtained points.

\section{Procedure}

Tests were administered in the school computer rooms in small groups (15 children each time). Order of administration was DiViSA first and afterwards TACI. The sample is described in terms of school years from $2^{\text {nd }}$ year until $6^{\text {th }}$ year. In Spain, $2^{\text {nd }}$ year corresponds to 6 years olds. For the same school year, the maximum difference in age between children is 11 months. 


\section{Results}

For each Test, seven variables were obtained ( 5 of them are the same for both tests). Reliability coefficients (Cronbach's alpha) were calculated for each variable and Test. For the DiViSA, reliability coefficients are high $(\alpha \geq .94)$ for the following variables: Global Attention Index, Commission Errors and Interval between Presses. For the variable Omission Errors, the obtained reliability coefficient is only sufficient but not good $(\alpha=.77)$ and the variables Attention Liability Between Items, Organization Index and Distraction-Precipitation Index do not show consistency. Reliability coefficients for all TACI variables are high: Global Attention Index ( $\alpha=$ .96), Commission Errors $(\alpha=.96)$, Interval between Presses $(\alpha=.93)$, Omission Errors $(\alpha=.95)$, Organization Index $(\alpha=.89)$, Performance Dis-inhibition Index $(\alpha=.93)$ and Learning Index $(\alpha=.95)$.

The obtained data with the DiViSA test about the developmental trend of attention show that, throughout the school years, the global attention index increases smoothly. At the same time, commission errors decrease until the fifth school year. The interval between presses decreases from second to third school year, then remains stable, and again decreases in the sixth school year. Attention liability between items, organization and distraction-precipitation indexes remain stable throughout the school years tested.

Obtained data with the TACI show that the global attention index increases progressively from second to sixth school year. Omission errors decrease slowly while commission errors remain stable. The organization index plays an important role in this Test; this index increases from second to fifth school year and afterwards remains stable.

\section{Discussion}

In our opinion, the two developed tasks to test attention in school children, from 6 to 12 years old, show some advantages to evaluate attention globally if compared with the available tests. If compared with paper and pencil tests, the new tests allow saving each child performance both on achievement and time variables as they are computerized. Both new tests require lower discrimination ability than others do and so, children as young as 6 years old can be tested and their performance in these new tests will not be confounded with their visual or cognitive abilities. If compared with the CPT (Continuous Performance Test), the new tests do not display the stimuli sequentially for a brief lapse but, each item includes all the stimuli spread over a field of information that include elements identical to the target and others different from it. This format fits the one usually employed for school work and, moreover, avoid confusions between omission errors due to blinking with true omission errors due to lack of attention. However, the elaboration of two new tests require additional studies as for instance, knowing the age range in which each test is most useful.

For both tests, obtained reliability coefficients are high. Next studies will focus on variable stability and DiViSA and TACI's convergent validity with other tests being used to measure attention in children.

Finally, the obtained developmental pattern is: (a) global attention index increases smoothly but constantly throughout the school years tested; (b) from the third school year, there are almost no commission errors if the task is a simple visual discrimination one while these errors decrease progressively from the second to the sixth school year if the task is more complex (c) omission errors decrease progressively, (d) the interval between presses decreases progressively only for the simple visual discrimination task. These results are consistent with the ones obtained by Klenberg et al. (2001), Lin et al., (1999) and Kanaka et al. (2008). Results from this paper show systematic age differences in the global attention index for both tasks but also show that continuity or discontinuity of the attention developmental pattern, previously obtained by other researchers, depends on task characteristics.

\section{Referencias}

American Psychiatric Association (2000). Diagnostic and statistical manual of mental disorders. 
Fourth edition. Text revision. Washington, DC, American Psychiatric Association. [Traducción al español: DSM-IV-TR (2002). Manual diagnóstico y estadístico de los trastornos mentales. Texto revisado. Barcelona: Elsevier Masson.]

Barkley, R. A. (1991). The ecological validity of laboratory and analogue assessment methods of ADHD symptoms. Journal of Abnormal Psychology, 19, 149-178.

Bennet, G., Seashore, H., y Wesman, A. (1990). DAT-5. Test de aptitudes diferenciales. Manual. Madrid: TEA.

Berger, A., Jones, L., Rothbart, M. K. y Posner, M. I. (2000). Computerized games to study the development of attention in childhood. Behavioral Research Methods and Instrumentation, 32, 290-303.

Brickenkamp R. (2002). D-2. Test de atención. Madrid: TEA Ediciones.

Castellanos, F. X., y Tannock, R. (2002). Neuroscience of attention deficit/ hyperactivity disorder: the search for endophenotypes. Nature Reviews Neuroscience, 3, 617-628.

Castillo, M. D. (2009). La atención. Madrid: Pirámide.

Cohen, J. (1988). Statistical power Analysis for the Behavioral Sciences. (2nd ed.). New Jersey: Lawrence Erlbaum Associates, Publishers.

Conners, C. K., Epstein, J. N., Angold, A., y Klaric, J. (2003). Continuous performance test performance in a normative epidemiological sample. Journal of Abnormal Child Psychology, 31 (5), 555-562.

Departamento I+D de TEA (2008). PMA. Test de Aptitudes Mentales Primarias. Madrid: Tea Ediciones S.A.

DSMIV-TR (2002). Manual diagnóstico y estadístico de los trastornos mentales. Texto revisado. Barcelona: Elsevier Masson.

Epstein, J. N., Erkanli, A., Conners, C. K., Klaric, J., Costello, J. E., y Angold, A. (2003). Relations between continuous performance test performance measures and ADHD behaviors. Journal of Abnormal Child Psychology, 31, 543-554.

Fan, J., McCandliss, B. D., Sommer, T., Raz, M. y Posner, M.I. (2002). Testing the efficiency and independence of attentional networks. Journal of
Cognitive Neuroscience, 14, 340-347.

García, E. M. y Magaz, A. (2000). Escala Magallanes de Atención Visual: EMAV. Bizkaia, España: Grupo ALBOR - COHS.

Gomez, R., Harvey, J., Quick, C., Scharer, I., y Harris, G. (1999). DSM-IV AD/HD: Confirmatory factor models, prevalence, and gender and age differences based on parent and teacher ratings of Australian primary school children. Child Psychology and Psychiatry, 40, 265-274.

Kanaka, N., Matsuda, T., Tomimoto, Y., Noda, Y., Matsushima, E., et al. (2008). Measurement of development of cognitive and attention functions in children using continuous performance test. Psychiatry and clinical Neurosciences, 62, 135141.

Klenberg, L., Korkman, M., y Lahti-Nuuttila, P. (2001). Differential development of attention and executive functions in 3- to 12-year-old Finnish children. Developmental Neuropsychology, 20, 407-428.

Korkman, M. (2001). Introduction to the special issue on normal neuropsychological development in the school-age years. Developmental Neuropsychology, 20, 325-330.

Lehman, E. B., Naglieri, J. A., y Aquilino, S. A. (2010). A national study on the development of visual attention using the cognitive assessment system. Journal of Attention Disorders, 14, 1524.

Lin, C. C. H., Hsiao, C. K. y Chen, W. J. (1999). Development of sustained attention assessed using the Continuous Performance Test among children 6-15 years of age. Journal of Abnormal Child Psychology, 27, 403-412.

López, J.A., Delgado, J., Pérez, I., Serrano, I., Alberola, S., Andrés, J.M., Sacristán, A. M., y Camina, A.B. (2010).Utilidad del Children's Embedded Figures Test en el Trastorno por Déficit de Atención con Hiperactividad. Clínica y Salud, 21, 93-103

McGee, R. A., Clark, S. E., y Symons, D. K. (2000). Does the Conners' Continuous Performance Test aid in ADHD diagnosis? Journal of Abnormal Child Psychology, 28, 415-424.

Naglieri, J.A. y Das, J.P. (1997). Cognitive Assessment system. Itasca, IL: Riverside. 
Posner, M. L., y Petersen, S. E. (1990). The attention system of the human brain. Annual Review Neuroscience, 13, 25-42.

Posner, M. I., y Raichle, M. E. (1994). Images of Mind. New York: Scientific American Library.

Rueda, M. R., Fan, J., McCandliss, B. D., Halparin, J. D., Gruber, D. B., Pappert Lercari, L., y Posner, M. I. (2004). Development of attentional networks in childhood. Neuropsychologia, 42, 10291040.

Santostefano, S. (1978). A biodevelopmental approach to clinical child psychology: cognitive controls and cognitive control therapy. New York: Wiley.

Santostefano, S. (1985). Cognitive Control Therapy with children and adolescents. Oxford, Pergamon Press. [Traducción: Terapia de Control Cognitivo con niños y adolescentes. Madrid: Ediciones Pirámide, S.A. 1990].

Santostefano, S. (1998). Handbook of Psychotherapy Integration. Nueva York: Jason Aronson.
Servera, M. y Llabrés, J. (2004). CSAT, Tarea de Atención Sostenida en la infancia. Madrid: TEA Ediciones S.A.

Thurstone, L. y Yela, M. (1985). Test de Percepción de Diferencias (Caras). Madrid: T.E.A. Ediciones.

Tudela, P. (1992). Atención. En J. L. F. Trespalacios y P. Tudela (eds), Atención y Percepción, (cap.4), Madrid: Alhambra.

Willcutt, E. G., Pennington, B. F., Boada, R., Ogline, J. S., Tunick, R. A., Chhabildas, N. A., y Olson, R. K. (2001). A comparison of the cognitive deficits in reading disability and attentiondeficit/hyperactivity disorder. Journal of Abnormal Psychology, 110 (1), 157-172.

Willcutt, E. G., Pennington, B. F., Olson, R. K., Chhabildas, N., y Hulslander, J. (2005). Neuropsychological analyses of comorbidity between reading disability and attention deficit hyperactivity disorder: in search of the common deficit. Developmental Neuropsychology, 27 (1), 35-78.

Manuscrito recibido: 07/09/2010

Revisión recibida: 22/11/2010

Manuscrito aceptado: 22/12/2010 
Anexo

Descriptores para la evaluación de la atención en TDAH según DSMIV-TR, índices que se obtienen del DiViSA-UAM y método para su cálculo

\begin{tabular}{|c|c|c|c|}
\hline $\begin{array}{l}\text { Conceptos } \\
\text { a evaluar } \\
\text { en TDAH }\end{array}$ & $\begin{array}{l}\text { Descriptores } \\
\text { DSM-IV-TR }\end{array}$ & $\begin{array}{l}\text { Índices obtenidos } \\
\text { en el DiViSA-UAM }\end{array}$ & $\begin{array}{c}\text { Cálculo } \\
\text { de los índices }\end{array}$ \\
\hline
\end{tabular}

\begin{tabular}{|c|c|c|c|}
\hline \multirow{7}{*}{ Desatención } & $\begin{array}{l}\text { Dificultades para mantener la atención } \\
\text { Atención insuficiente a detalles }\end{array}$ & $\begin{array}{l}\text { Índice de atención global } \\
\text { Errores de comisión }\end{array}$ & $\begin{array}{l}\left(\mathrm{N}^{0} \text { de aciertos }-\mathrm{N}^{\circ} \text { de errores }\right) / \text { Tiempo } \\
\text { Pulsaciones realizadas fuera de un árbol } \\
\text { modelo }\end{array}$ \\
\hline & Ensimismamiento, retirada de atención a la tarea & Intervalo entre pulsaciones. & $\begin{array}{l}\text { Duración del ensayo / } \mathrm{N}^{\circ} \text { total de pulsacio- } \\
\text { nes }\end{array}$ \\
\hline & Labilidad atencional & Labilidad inter-ensayos & $\begin{array}{l}\text { Varianza inter-ensayos del índice de aten- } \\
\text { ción global }\end{array}$ \\
\hline & Olvidos & Errores de omisión & $\begin{array}{l}\mathrm{N}^{\circ} \text { de árboles iguales al modelo que en } \\
\text { cada matriz se deja sin pulsar }\end{array}$ \\
\hline & Desagrado y aversión ante el esfuerzo & & \\
\hline & Distracción ante lo irrelevante & & \\
\hline & Desorganización & Índice de organización & $\begin{array}{l}\text { Secuencia y orden en que se recorre la pan- } \\
\text { talla pulsando sobre las figuras }\end{array}$ \\
\hline \multirow[t]{2}{*}{ Impulsividad } & Precipitación al responder & Índice de Distracción-precipitación & $\begin{array}{l}\text { Tiempo de respuesta (TR) en las pulsacio- } \\
\text { nes incorrectas menos TR en las pulsacio- } \\
\text { nes correctas (TRE-TRA) }\end{array}$ \\
\hline & Dificultad para esperar & No calculable & \\
\hline
\end{tabular}

Descriptores para la evaluación de la atención en TDAH según DSMIV-TR, índices que se obtienen del TACI-UAM y método para su cálculo

\begin{tabular}{|c|c|c|c|}
\hline $\begin{array}{l}\text { Conceptos } \\
\text { a evaluar } \\
\text { en TDAH }\end{array}$ & $\begin{array}{l}\text { Descriptores } \\
\text { DSM-IV-TR }\end{array}$ & $\begin{array}{l}\text { Índices obtenidos } \\
\text { en el TACI-UAM }\end{array}$ & $\begin{array}{c}\text { Cálculo } \\
\text { de los índices }\end{array}$ \\
\hline \multirow{8}{*}{ Desatención } & Dificultades para mantener la atención & Índice de atención global & $\begin{array}{l}\left(\mathrm{N}^{o} \text { de aciertos - } \mathrm{N}^{\circ} \text { de errores }\right) / \mathrm{n}^{\circ} \text { de ensa- } \\
\text { yos }\end{array}$ \\
\hline & Atención insuficiente a detalles & Errores de comisión & $\begin{array}{l}\mathrm{N}^{\circ} \text { de pulsaciones con las que no se obtie- } \\
\text { nen } 9 \text { puntos }\end{array}$ \\
\hline & Ensimismamiento, retirada de atención a la tarea & Intervalo entre pulsaciones. & $\begin{array}{l}\text { Duración del ensayo ( } 43 \mathrm{~s}) / \mathrm{N}^{\circ} \text { total de pul- } \\
\text { saciones }\end{array}$ \\
\hline & Labilidad atencional & No calculable & \\
\hline & Olvidos & Errores de omisión & Número de fichas de 9 puntos no pulsadas. \\
\hline & Desagrado y aversión ante el esfuerzo & & \\
\hline & Distracción ante lo irrelevante & & \\
\hline & Desorganización & Índice de organización & $\begin{array}{l}\text { Secuencia y orden con que se recorre la } \\
\text { pantalla pulsando sobre las figuras de cua- } \\
\text { drúpedos }\end{array}$ \\
\hline \multirow{3}{*}{ Impulsividad } & Precipitación al responder. & No calculable en la versión actual & \\
\hline & Dificultad para esperar & Control de la actuación (inhibición) & $\begin{array}{l}\mathrm{N}^{\circ} \text { de pulsaciones en situación de espera o } \\
\text { prohibición (pantalla en amarillo) }\end{array}$ \\
\hline & & Aprendizaje & $\begin{array}{l}\text { Proporción de pulsaciones sobre figuras de } \\
9 \text { puntos / total de figuras pulsadas }\end{array}$ \\
\hline
\end{tabular}

